\title{
Assessing the Human Factors risks in extending the use of AWS
}

\author{
Ronald W. McLeod ${ }^{1}$, Guy H. Walker ${ }^{2}$ and Ann Mills ${ }^{3}$
}

\author{
${ }^{1}$ Nickleby HFE Ltd, Hillington Park Innovation Centre, Glasgow, G52 4RU \\ ${ }^{2}$ Brunel University, Ergonomics Research Group, Uxbridge, UB8 3PHH \\ ${ }^{3}$ Rail Safety \& Standards Board, Euston, London, NW1 2DX
}

Keywords: AWS, SPAD, Mode Errors; Driver reliability; risk assessment

\begin{abstract}
The project reported in this paper was conducted on behalf of the Rail Safety and Standards Board, and formed part of the SPAD reduction and mitigation research theme. It sought to assess the Human Factors risks associated with extending the use of the in-cab Automatic Warning System (AWS). The term "Extended AWS" refers to any situation where AWS is used other than to warn of the state of upcoming signals. This includes uses for permanent, temporary and emergency speed restrictions, certain level crossings, and, potentially, multi-SPAD signals. The paper summarises the work performed in the study. It considers new areas of psychological investigation believed to be important for driver related research, the methods used to gather and analyse industry experience, and concludes by examining the risk of drivers failing to behave appropriately to AWS warnings.
\end{abstract}

\section{The Problem}

AWS was originally intended to support semaphore signalling, and only later became extensively used within three and four aspect colour light signalling. However, it only provides warnings that discriminate between two signal states: clear (green, indicated by a bell or simulated chime, at $1200 \mathrm{~Hz}$ ) and caution (red, single or double yellow, indicated by a steady alarm or horn sound at $800 \mathrm{~Hz}$ ).

AWS is intended as a supporting system. The driver overrides the automatic application of the brakes by acknowledging the audible warning (horn) and retains manual control of the train. Cancelling a caution (horn) initiates a visual reminder in the form of the 'sunflower' on the driver's desk, indicating that the last signal passed was showing caution (or even stop). The maintenance of safety therefore depends, fundamentally, on the reliability of the driver.

Describing the 1936 Strowger-Hudd system adopted by BR as AWS, Vaughan [1] writes that; "No-one ever thought that the driver would ignore the warning. It was 
utterly taken for granted that a driver would always take notice of the signal. (p.12). In connection with the crash at Watford Junction in 1996, Stanley Hall [2], another very experienced railwayman turned author states; "It seems inconceivable that a driver can acknowledge receiving two warnings and yet take no action to apply the brake to stop the train at the red signal, yet it happens". (p.97). This paradox provided the background and context for the study; despite what, to all everyday experience, appears to be an extremely powerful attention-getting device, combined with a highly visible visual reminder, there is a significant, and recurring risk of AWS failing to prevent experienced train drivers passing signals at danger.

There were three main study objectives. The first was to investigate whether extended AWS could, or already has, led to a change in the risk of driver unreliability, potentially leading to Signals Passed At Danger (SPAD). The second objective was to understand the circumstances in which further extending the use of AWS might reduce driver reliability. Finally, the study was required to provide a basis for predicting situations in which driver reliability with AWS might be significantly reduced. The overall aim was to provide a basis for understanding the implications of further extending the use of AWS to, for example, forewarn train drivers of approaching signals that have a history of multiple SPADs. (The study was specifically not required to consider the desirability or feasibility of any technical changes to current AWS equipment, such as additional tones or indications.)

\section{Industry Data}

Field research sought to capture the extensive operational experience available within the industry, and to gather detailed insights on the possible influences that might lead to driver errors with (or despite) AWS. Full details of this survey of industry are reported in [3]. The information was gathered using focus group methods, cab-rides, a questionnaire survey of 277 drivers, a sample of OTMR (OnTrain-Monitoring and Recording) data, and incident data from SMIS and CIRAS. The results of this exercise have been validated by industry stakeholders.

\section{Extended AWS and SPAD risk}

In general, drivers expressed very positive views towards AWS as an aid to safe driving. Drivers report that the obvious limitations of the system (e.g. the simple two state warnings) are largely overcome through the use of defensive driving techniques. Drivers also express confidence with the way in which AWS is currently used, and suggested that they would consider the network less safe if AWS was restricted solely to signal aspects (i.e. no extended AWS at all). Despite this, there are clearly many occasions where drivers experience doubt, confusion, or uncertainty about the meaning of AWS warnings associated with extended usage.

In our questionnaire survey of 277 drivers, more than three-quarters reported having experienced an AWS magnet that they were not expecting. $18 \%$ of these unexpected magnets were due to extended AWS usage for Temporary Speed Restrictions (TSR). Similarly, 16\% of drivers report having experienced confusion 
about the meaning of an AWS warning and 34\% of these sites were for extended AWS. When asked to suggest what factors are likely to increase risk with AWS usage, Emergency Speed Restrictions (ESR), Temporary Speed Restrictions (TSR), and Permanent Speed Restrictions (PSR) (all extended uses) were placed in first, second, and third places respectively. The findings from this survey clearly indicate that extended AWS has and does potentially increase the risk of SPADs.

\section{AWS and driver behaviour}

Results from the driver questionnaire survey show that a significant number of drivers (155 out of 277 , or $56 \%$ ) report having 'automatically' cancelled an AWS warning on one or more occasions. As Figure 1 shows, the great majority state that they have cancelled AWS automatically between 1 and 6 times (i.e. a few times). Interestingly, a very small percentage (2\%) report that they experience this on a daily basis. Once again, extended AWS issues (for example, "When the relevance of the horn for a TSR seems a little less important than coming up to restrictive signals") accounted for around $20 \%$ of the reasons given by drivers for automatic AWS cancellations. Personal factors, the quantity of warnings, and signalling issues (such as prolonged running on cautions) also feature prominently.

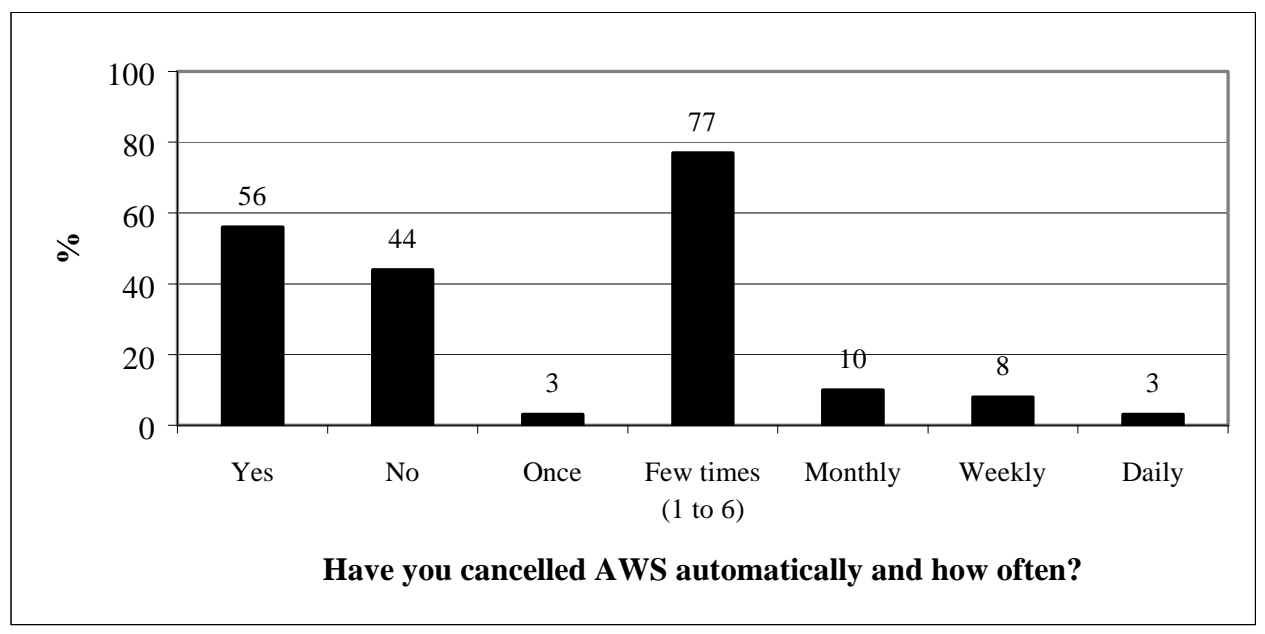

Figure one: Responses to question 4, "Have you ever experienced cancelling AWS automatically".

A small sample of OTMR data was made available to the study, and although limited to the extent that it merely provides a snapshot of a specific day, driver, driving style and route, it does offer some objective indication of how drivers react to AWS. (A larger scale analysis was beyond the scope and resources of the project). Figure 2 illustrates that on seven out of eleven occasions where an AWS horn indication was received, the driver was operating the cancellation button before the horn had started to sound. This seems to suggest that the driver's response was anticipatory and in response to sight of the AWS track magnet ahead. On occasions where the driver receives the horn and then responds the average response time calculated from the OTMR data was 0.60 seconds (minimum time is 0.49 seconds, 
maximum time is 0.89 seconds). The speed of this response time again suggests that the cancellation behaviour is highly learnt, and/or anticipatory in nature.

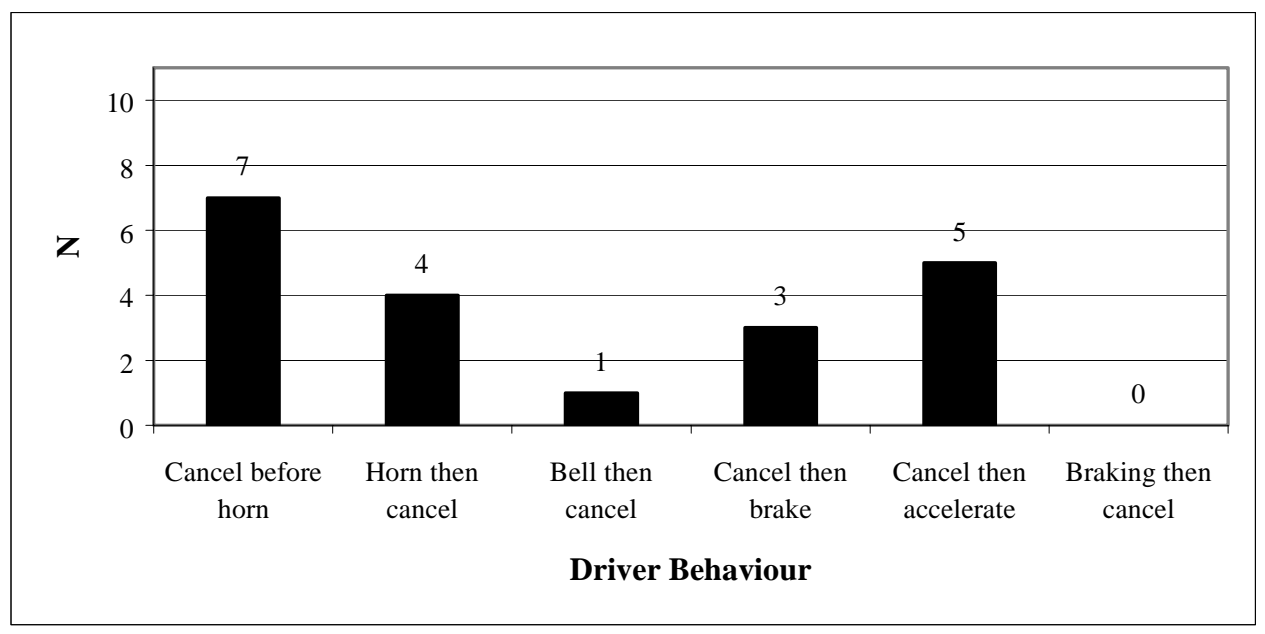

Figure two: Driver behaviour in response to AWS horn

The OTMR data confirms that there is no single, fixed behavioural response expected of a driver when in receipt of an AWS warning. For three of the AWS cancellation events illustrated in Figure 2 above, the immediate behaviour was to begin braking, but on five out of 11 occasions the AWS cancellation was followed or accompanied by accelerating the train. Although seemingly counter to the explicit purpose of AWS (which is to prompt the driver to the possible need to slow down) this behaviour is for the most part entirely appropriate and legitimate. Another interesting aspect of the data is that there are no instances of the AWS horn occurring after the driver has initiated a braking manoeuvre. That is, situations where the driver had sighted the signal and has started to respond in advance of the AWS activation. Behaviour of this sort would be expected to be reasonably frequent. It is not clear why it was not seen in this sample of OTMR data.

\section{Mode Errors}

Many factors specific to the driver, the class of rolling stock involved, the nature of the movement, and the situation at the time the warning occurs will determine how and when an individual driver reacts to an AWS warning. As Woods [4] notes, this situation "shifts to a reliance on human adaptability to make [AWS] work (in principle people can keep track of the context) [...] but sometimes they will misinterpret in circumstances where it matters". This misinterpretation is defined as a mode error, with the result that the likelihood of a SPAD is significantly increased. To reinforce this point, SMIS data (covering the period 1996 to 2002) defines the most common cause of a SPAD as being where a driver fails to respond to a cautionary signal. In these situations the driver correctly cancels the AWS warning, but misinterprets the circumstances (i.e. commits a mode error) and does not react with appropriate action to brake the train. The industry data presented above provide insight into why, and possibly when such situations may arise. 


\section{Assessment of driver reliability with Extended AWS}

There was no requirement in the AWS study to attempt to quantify the absolute level of risk - in terms of the likelihood of occurrence - associated with Extended AWS. However, in common with established safety management practice, the assessment sought to establish whether the level of risk associated with extended uses of AWS is "as low as is reasonably practical" (ALARP).

\section{Analysis method}

The approach used to assess the risk of driver unreliability associated with extended AWS was based on consideration of the likely psychological mechanisms and constructs involved in influencing driver behaviour. This included a "Situational Model” of train driver performance. The background thinking is summarised in [6]. The approach emphasises the need to try to understand the driver's possible psychological state at the time at which warnings are current. The analysis method involved the following steps:

1. A set of twenty AWS usage scenarios were developed. These were based largely on discussions with drivers, and are thought to be representative of many situations encountered across the UK rail network. These scenarios are summarised on table 1.

2. Each scenario was presented graphically along a timeline (the time dimension of the evolution of risk with AWS has emerged as possibly the key situational factor).

3. By consideration of the way in which each scenario develops over time, situational factors considered likely to be 'active' at each point in the scenario were identified and mapped onto the graphical presentation. These were drawn from a set of eighteen potential AWS specific situational factors generated from information gathered in the industry survey.

4. A very simple influence model was used to explicitly represent the way in which the situational factors might affect driver reliability with AWS. This model is encapsulated in the following two simple assumptions. First, if any of the situational factors are identified as being likely to be present at the time that an AWS warning is active, there will be an increased risk of the driver making a mode error. Second, the more factors present at the time an AWS warning is active, the greater the risk of driver unreliability. No attempt was made to account for interactions between the situational factors, or cumulative effects if multiple factors might be active. 
Table one: Brief description of the twenty AWS usage scenarios

\begin{tabular}{|c|c|}
\hline Scenario & Brief Description \\
\hline 1 & $\begin{array}{l}\text { Incremental increases in line speed which do not reflect actual train acceleration. PSR } \\
\text { and attendant AWS redundant, and in close proximity to signal. }\end{array}$ \\
\hline 2 & $\begin{array}{l}\text { Driver already braking for signal when AWS received for PSR. Driver responds to line } \\
\text { speed increases instead of AWS indications for cautionary signal. }\end{array}$ \\
\hline 3 & $\begin{array}{l}\text { Driver is braking appropriately in response to AWS for TSR, but not appropriately for } \\
\text { upcoming danger signal. TSR commencement is beyond signal at danger. }\end{array}$ \\
\hline 4 & $\begin{array}{l}\text { Four aspect signalling interspersed with PSR's. Driver is distracted by guard } \\
\text { communication while receiving AWS warnings in quick succession for signal and PSR. }\end{array}$ \\
\hline 5 & $\begin{array}{l}\text { Interrupted view of facing signals on bi-directional line, driver expects certain aspect(s) } \\
\text { to be displayed. AWS does not discriminate between signals at caution or danger. }\end{array}$ \\
\hline 6 & $\begin{array}{l}\text { Multiple AWS warnings due to trains bunching in rear of ESR. Repetitive AWS } \\
\text { cancellations with no action required as train speed is already low. }\end{array}$ \\
\hline 7 & $\begin{array}{l}\text { Closely spaced AWS magnets for PSR and signal. Driver receives horn but sunflower } \\
\text { remains black, or, receives continuous horn activation requiring one cancellation not } \\
\text { two. }\end{array}$ \\
\hline 8 & $\begin{array}{l}\text { Sunflower left activated after departing from AWS-fitted route onto an unfitted route. } \\
\text { TSR in place with AWS, sunflower remains active for TSR and last fitted signal. }\end{array}$ \\
\hline 9 & $\begin{array}{l}\text { Driver receives AWS at distant signal. Driver subsequently departs from station with } \\
\text { sunflower active, proceeds through successive stop signals, but last in sequence is } \\
\text { showing danger. }\end{array}$ \\
\hline 10 & $\begin{array}{l}\text { Driver reads-through to signal on wrong line. Correct AWS indication is assumed to } \\
\text { be a Code } 2 \text { fault. }\end{array}$ \\
\hline 11 & $\begin{array}{l}\text { Single line working with unsuppressed magnets. Initial reaction to AWS warnings is } \\
\text { that they are redundant even though they may apply to an unexpected stop aspect. }\end{array}$ \\
\hline 12 & $\begin{array}{l}\text { TSR encountered by freight driver on } 125 \mathrm{mph} \text { line. Sunflower already active due to } \\
\text { cautionary signals. Driver forgets TSR within lengthy } 125 \mathrm{mph} \text { braking distance. }\end{array}$ \\
\hline 13 & AWS magnet missing. Driver expecting warning, none received, braking occurs late. \\
\hline 14 & $\begin{array}{l}\text { AWS magnet buried by track ballast. Driver using defensive driving techniques, } \\
\text { cannot site magnet which leads to late braking. }\end{array}$ \\
\hline 15 & $\begin{array}{l}\text { Signal located in tunnel. AWS coincides with having to sound horn and other train } \\
\text { approaching. Poorly sited sunflower combined with AWS gap area. }\end{array}$ \\
\hline 16 & $\begin{array}{l}\text { Driver passes signal at danger. TPWS activates, driver does not notice TPWS light. } \\
\text { Assumes problem is an unsolicited AWS brake demand, resets and departs. }\end{array}$ \\
\hline 17 & $\begin{array}{l}\text { Competing priorities (view of signal and responding to AWS versus safety of track } \\
\text { workers). }\end{array}$ \\
\hline 18 & $\begin{array}{l}\text { Confusing TSR layout. Driver cancelling AWS just to keep the brakes off due to } \\
\text { insufficient time to attribute individual warnings to specific signage. }\end{array}$ \\
\hline 19 & $\begin{array}{l}\text { Driver braking for station when AWS received for ABCL crossing. Distractions during } \\
\text { station stop, driver attributes sunflower to next signal not to level crossing. }\end{array}$ \\
\hline 20 & $\begin{array}{l}\text { Change in meaning of AWS referent. AWS refers to spate board, spate board then } \\
\text { missing so AWS warning is a Code } 8 \text { fault, same AWS then changed to active TSR. }\end{array}$ \\
\hline
\end{tabular}




\section{Analysis Example}

An example of an analysis is shown below in figure three. The scenario develops from left to right. The critical point exists between points A and B. Here the driver has accelerated to 60mph and as usual does not need to take any action for the PSR (at point 6) because trains cannot reach the previous line speed of $90 \mathrm{mph}$. From point 6 onwards the active sunflower is ambiguous because it refers to the unusual cautionary signal aspect encountered at point 5, and the usual redundant PSR encountered at point 6. Between points A and B six situational factors are considered as potentially active. Using the simple influence model, this combination of factors clearly offers the potential for the driver to make a 'mode error', by confusing the meaning of the sunflower and not applying the brake in time for the upcoming signal at danger.

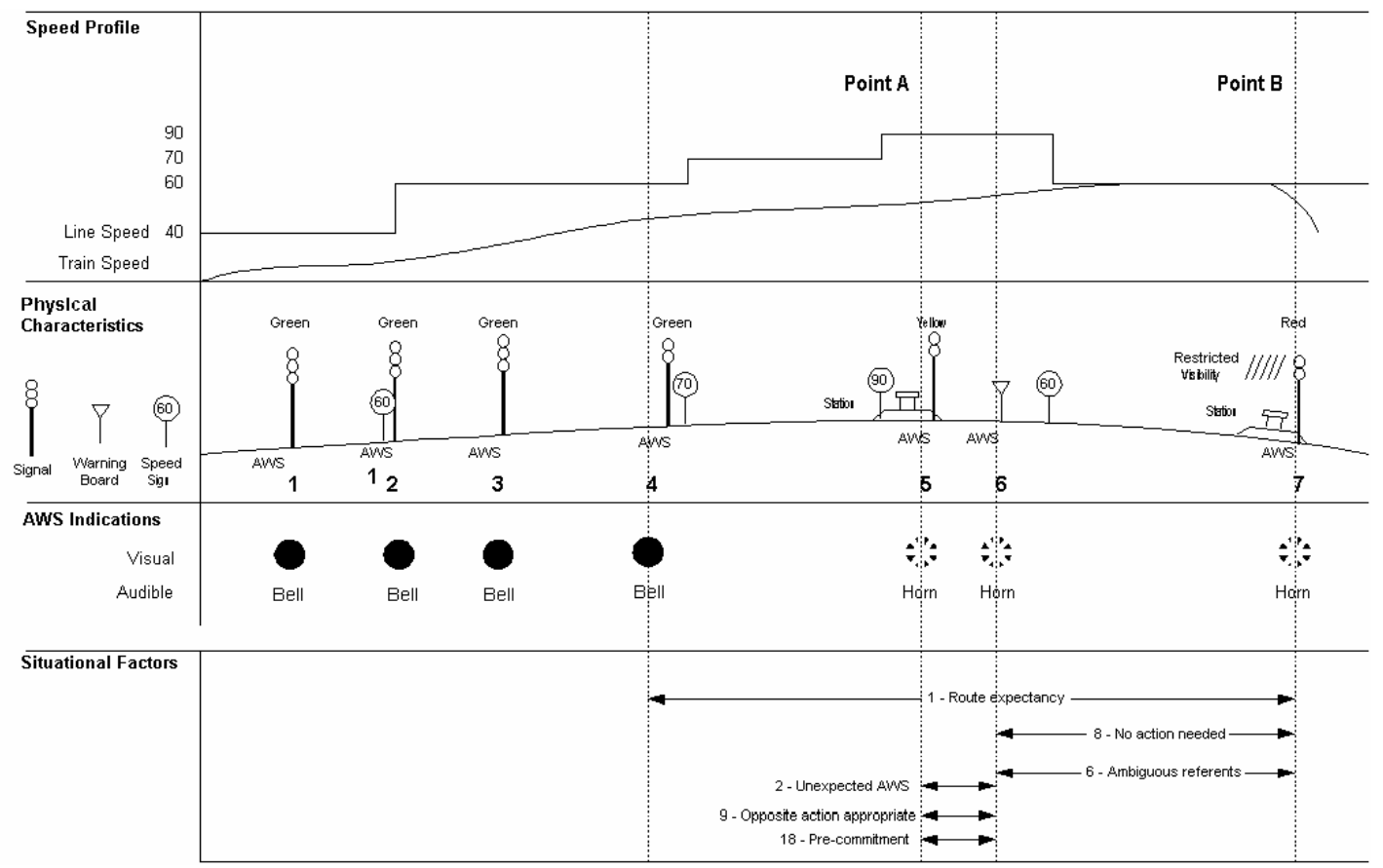

Figure three: Graphical representation of scenario 1 of 20

In this case there are a number of actions that could potentially be taken which would align the scenario with ALARP principles. These include reducing the line speed in order to reflect the accelerative abilities of trains using the route, and thus doing away with a reduction in speed that necesitates an AWS installation. Alternatively, the PSR could be enforced using the existing practice of approach release signalling, or using TPWS in a new way to provide protection against overspeeding without the need for an overt AWS warning. These possible solutions 
would mean that the sunflower refers to only one source, therefore reducing ambiguity, as well as ensuring that route characteristics (or driveability) matches appropriate driver behaviour more closely.

\section{Overall analysis results}

Figure four below presents a summary of the number of times that each of the eighteen situational factors apply within all the twenty AWS usage scenarios. Four situational factors are identified most frequently, these are; route expectancy (expectations about the route ahead), uncertainty (over what a warning refers to), ambiguous AWS referents (where the sunflower refers to more than one thing), and no action needed (in response to a warning). Consideration of these scenarios suggests that there are a number of actions that are both reasonable and practical that could potentialy be taken to reduce the risk to a level which is ALARP.

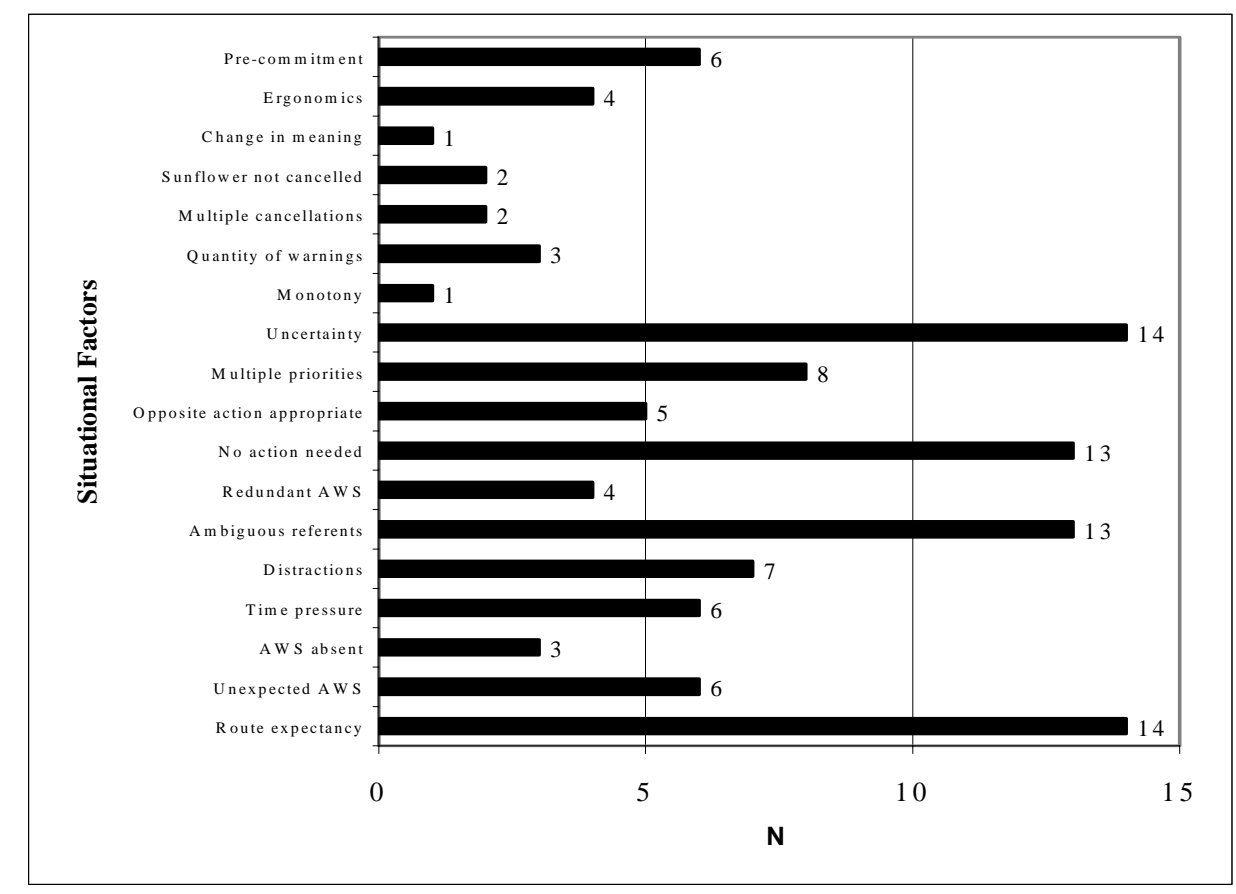

Figure four: Summary of situational factors associated with the 20 scenarios

Potential interventions that might help reduce the risk of driver unreliability with extended AWS include;

- $\quad$ ensuring that the AWS referent is clear and unambiguous

- ensuring that the implementation of TSR/ESRs follows standardised practice

- designing line speed profiles that approximate to the accelerative and speed capabilities of stock using the route (to avoid redundant drops in line speed and AWS) 
- providing incremental drops in line speed instead of large decrements that require an AWS wanring (also consider incremental drops in speed in advance of an ESR to lessen the effects of bunching traffic)

- in some cases consider imposing a different ESR speed to that normally required for running under single yellow aspects (this would help break the routine of AWS cancellation followed by no action needed)

- $\quad$ employing TPWS (or TPWS+) as the primary safety device at PSRs instead of AWS

- considering the acceptable spacing distance for signals and static AWS warnings for PSRs

- controlling the speed of approaching trains with approach release signals

- considering the use of reminder signs (e.g. for active speed restrictions) at critical decision points in a situation

- considering the position of TSR/ESR termination boards in relation to signals (to ensure that the mode change from multiple to single AWS referents does not cause confusion)

- $\quad$ reconsidering the use of AWS at spate boards.

\section{Conclusions}

The study set out to understand and assess the risks of driver unreliability associated with Extended AWS. There were three objectives;

\section{Objective 1: Has extended AWS increased risk}

The answer to the first objective is clearly "Yes". The evidence strongly suggests that extended uses of AWS should be assumed to have increased the risk of driver unreliability. There are four principal reasons for this;

- the same AWS warning can refer to any of a number of different states. Other than the driver's own awareness and route knowledge, there is nothing that discriminates between the different sources.

- multiple AWS warnings, with different sources, are frequently current simultaneously. There is therefore a reliance on the driver's memory not only to know how many warnings are current, but what they refer to.

- the sunflower is inherently ambiguous: it does not discriminate between the source of the last AWS warning, but simply indicates whether the last warning was a horn or a bell.

- $\quad$ because of the variety of causes, extended AWS has many more implications for driver behaviour than was originally the case.

These four factors potentially increase the probability of the driver misinterpreting the circumstances and making a mode error. 


\section{Objective 2: the circumstances leading to driver unreliability}

A key premise underlying the study has been that driver performance with AWS can only be understood in terms of the context and situation at the time the driver encounters a warning. In particular, the events immediately preceding and coincident with the warning, and the driver's expectations about the route ahead, are critically important.

Because of this, the response to the second objective is less conclusive than for the first. In general, given the controls and procedures that are already in place, there are unlikely to be easily identifiable circumstances inherent in a particular AWS site that would tend to lead to driver unreliability. Essentially, it would be incorrect to view an AWS site itself as being inherently high or low risk (assuming it has been implemented and maintained in accordance with Group Standards). The conclusion from this study is that it would only be correct to consider the likelihood of a driver encountering an AWS warning under conditions which cause one or more of the situational factors influencing driver behaviour to be active.

\section{Objective 3: a predictive method}

The third objective of the study was to provide a basis for predicting situations in which the risk of driver reliability with AWS is likely to be significantly reduced. The analysis method developed in the study and used to analyse the twenty AWS usage scenarios provides such a basis.

Further validation of the method is likely to be required to improve its predictive power and validity. In particular, further development is required to represent the combined effect when multiple situational factors are thought likely to be present simultaneously.

The method may be considered to have greater power as a retrospective analysis tool, for example in SPAD investigations; i.e. in seeking to identify the factors that led to an incident which has already occurred. The method has significant potential to help avoid simplistic conclusions such as "driver inattention", and to help identify what might have gone on "in the drivers head" before an incident. As it stands, the method is thought to provide a reasonably structured approach to reviewing AWS sites with a view to identifying the potential for driver unreliability.

\section{References}

1. Hall, S. (1999). Hidden dangers. Railway safety in the era of privatisation. Shepperton, Surrey: Ian Allen.

2. Vaughan, A. (2000). Tracks to disaster. Shepperton, Surrey: Ian Allen.

3. Walker, G. H., \& McLeod, R. W. (Jul-2003). Extended AWS study: Report on industry data. Nickleby HFE Ltd.

4. Woods, D. D. (May, 2003). Personal communication 
5. McLeod, R. W., \& Walker, G. H. (Aug-2003). Assessment of driver reliability with extended AWS. Nickleby HFE Ltd.

6. McLeod, R. W., Walker, G. H., \& Moray, N. (Jul-2003). Extended AWS study: Review of the knowledge base. Nickleby HFE Ltd. 